\title{
Potential Effectiveness of Bromhexine Hydrochloride as an Affordable Over-the-counter Drug for Prophylaxis Against COVID-19 in Developing Countries
}

\author{
Milad Shirvaliloo ${ }^{1}$ and Roghayeh Sheervalilou ${ }^{2}$ \\ ${ }^{1}$ Tabriz University of Medical Sciences \\ ${ }^{2}$ Zahedan University of Medical Sciences
}

June 15,2020

\section{Dear Editor,}

Since the early 2020, many countries around the world have become afflicted with COVID-19, a respiratory infection caused by SARS-CoV-2, that rapidly turned into an overwhelming pandemic, comparable to devastating outbreaks such as the Spanish flu. As the well-funded investigational efforts to develop preventive strategies and therapeutic agents continues in the developed countries, new concerns regarding the prospective waves of coronavirus infection arise in the developing countries, which simply do not have access to effective treatments that have been adopted in the modern world. However, based on the most recent evidence regarding the pathogenesis of SARS-CoV-2, there is hope that certain cost-effective medications, such as bromhexine hydrochloride, might actually prove useful in containment of COVID-19, either as an outpatient treatment or a prophylactic measure.

Shortly after the publicity of COVID-19 outbreak, numerous investigations were conducted on almost every aspect of the novel coronavirus, particularly the pathogenesis of SARS-CoV-2. A number of studies reported that the virus made its entry into the cell by means of Transmembrane Serine Protease 2 (TMPRSS2), among several other receptors, most notably, Angiotensin Converting Enzyme 2 (ACE2) (Hoffmann et al., 2020). This was mostly similar to the entry mechanism adopted by SARS-CoV and MERS-CoV, as both strains were reported to have interactions with TMPRSS2, which ultimately resulted in the entry of the virus to the susceptible host cell, following the activation of Spike or S protein of the virus (Shen, Mao, Wu, Tanaka, \& Zhang, 2017). Similar results involving TMPRSS2 have also been documented with VeroE6 cells, as expression of TMPRSS2 in these cells rendered them highly susceptible to SARS-CoV-2 infection (Matsuyama et al., 2020).

It is speculated that ACE2 and TMPRSS2 function together in favor of SARS-CoV-2 cell entry, with the former binding to the $\mathrm{S}$ protein on the virion, and the latter priming it, so that the virion can enter the cell. During the priming, TMPRSS2 cleaves the S protein at the S1/S2 arginine-rich site of the viral protein. However, the action of TMPRSS2 does not seem to be solely restricted to processing of the S protein, as it was indicated that this serine protease might also be involved in regulation of the assembly of viral particles in the Golgi apparatus, before the virions can be expelled from the cell (Hoffmann et al., 2020). Investigations on the expression profile of TMPRSS2 and ACE2 in the bronchial and alveolar tissue of the lung indicated that TMPRSS2 can be found in both cell types, however, ACE2 is chiefly produced by transient secretory cells located in the subsegmental bronchial epithelium (Lukassen et al., 2020). Since it is more widely distributed in the lung, TMPRSS2 can be regarded as a key factor involved in the pathogenesis of SARS-CoV-2, which 
can be duly inhibited by specific drugs that have been in use for a long time, particularly bromhexine hydrochloride.

Most commonly recognized as an expectorant or cough syrup, bromhexine hydrochloride is a mucolytic drug known to many communities since its introduction back in 1963. As the drug class "mucolytic" might suggest, bromhexine is often prescribed for treatment of illnesses associated with excessive production of phlegm, as a result of disturbances in normal mucous secretion by the secretory cells in the epithelium of the bronchi. An investigation in 2014 on prostate cancer metastasis revealed that bromhexine hydrochloride possessed certain properties that made it a potent inhibitor of TMPRSS2, selectively targeting the transmembrane serine protease 2 with an $\mathrm{IC}_{50}$ of $0.75 \mu \mathrm{M}$ (Lucas et al., 2014). This finding is of crucial significance now, as TMPRSS2 is widely expressed in the lung, and has been indicated to be involved in the cell entry of the novel coronavirus (Lukassen et al., 2020).

As a mucolytic drug, bromhexine hydrochloride comes with several important advantages. It is an overthe-counter (OTC) drug, that can be purchased without a prescription. It is an affordable medication, that is well tolerated by most of the patients, and has not been known to cause any major adverse effects (Depfenhart, de Villiers, Lemperle, Meyer, \& Di Somma, 2020).

As Dr. Tedros Adhanom Ghebreyesus, World Health Organization (WHO) Director-General, said "Pandemic is not a word to use lightly or carelessly." The COVID-19 pandemic has indeed posed a great challenge to the entire world, but more importantly, the developing countries and the less fortunate communities, that are struggling to contain the insidious outbreak. Due to the scarcity of the recently developed treatments and also the long-known expensive medications, it is totally clear that cheaper and more affordable methods should be adopted by the underdeveloped countries. With due attention to the safety of bromhexine hydrochloride, it might be a reasonable strategy to adopt this medication for outpatient management of patients with mild disease, or even advise to use it as a prophylactic measure to communities that might be in great risk. It must be clarified that despite the evidence discussed in this paper, precise clinical trials are required to appraise the effectiveness of this long-known mucolytic drug in treatment of COVID-19, and potentially prophylaxis against it.

Keywords : Bromhexine, SARS-CoV-2, TMPRSS2, ACE2

\section{Conflict of Interest Statement}

All authors involved in this work declare that they have no conflict of interest.

\section{References}

Depfenhart, M., de Villiers, D., Lemperle, G., Meyer, M., \& Di Somma, S. (2020). Potential new treatment strategies for COVID-19: is there a role for bromhexine as add-on therapy? Internal and Emergency Medicine . doi:10.1007/s11739-020-02383-3

Hoffmann, M., Kleine-Weber, H., Schroeder, S., Krüger, N., Herrler, T., Erichsen, S., . . Pöhlmann, S. (2020). SARS-CoV-2 Cell Entry Depends on ACE2 and TMPRSS2 and Is Blocked by a Clinically Proven Protease Inhibitor. (1097-4172 (Electronic)).

Lucas, J. M., Heinlein, C., Kim, T., Hernandez, S. A., Malik, M. S., True, L. D., . . Nelson, P. S. (2014). The Androgen-Regulated Protease TMPRSS2 Activates a Proteolytic Cascade Involving Components of the Tumor Microenvironment and Promotes Prostate Cancer Metastasis.Cancer Discovery, 4 (11), 1310. doi:10.1158/2159-8290.CD-13-1010

Lukassen, S., Chua, R. L., Trefzer, T., Kahn, N. C., Schneider, M. A., Muley, T., . . Eils, R. (2020). SARSCoV-2 receptor ACE2 and TMPRSS2 are primarily expressed in bronchial transient secretory cells. The EMBO Journal, 39 (10), e105114. doi:10.15252/embj.20105114

Matsuyama, S., Nao, N., Shirato, K., Kawase, M., Saito, S., Takayama, I., . . Takeda, M. (2020). Enhanced isolation of SARS-CoV-2 by TMPRSS2-expressing cells. Proceedings of the National Academy of Sciences, 
117 (13), 7001. doi:10.1073/pnas.2002589117

Shen, L. W., Mao, H. J., Wu, Y. L., Tanaka, Y., \& Zhang, W. (2017). TMPRSS2: A potential target for treatment of influenza virus and coronavirus infections. Biochimie, 142 , 1-10. doi:https://doi.org/10.1016/j.biochi.2017.07.016 